\title{
Smart photogalvanic running-grating interferometer
}

Kukhtarev, N. V.; Kukhtareva, T.; Edwards, M. E.; Jones, J.; Bayssie, M.; Wang, J.; Lyuksyutov, S. F.; Reagan, M. A.; Buchhave, Preben

Published in:

Journal of Applied Physics

Link to article, DOI:

$10.1063 / 1.1850171$

Publication date:

2005

Document Version

Publisher's PDF, also known as Version of record

Link back to DTU Orbit

Citation (APA):

Kukhtarev, N. V., Kukhtareva, T., Edwards, M. E., Jones, J., Bayssie, M., Wang, J., Lyuksyutov, S. F., Reagan, M. A., \& Buchhave, P. (2005). Smart photogalvanic running-grating interferometer. Journal of Applied Physics, 97(5), 054301. https://doi.org/10.1063/1.1850171

\section{General rights}

Copyright and moral rights for the publications made accessible in the public portal are retained by the authors and/or other copyright owners and it is a condition of accessing publications that users recognise and abide by the legal requirements associated with these rights.

- Users may download and print one copy of any publication from the public portal for the purpose of private study or research.

- You may not further distribute the material or use it for any profit-making activity or commercial gain

- You may freely distribute the URL identifying the publication in the public portal

If you believe that this document breaches copyright please contact us providing details, and we will remove access to the work immediately and investigate your claim 


\title{
Smart photogalvanic running-grating interferometer
}

\author{
N. V. Kukhtarev, T. Kukhtareva, M. E. Edwards, J. Jones, M. Bayssie, and J. Wang \\ Department of Physics, Alabama A\&M University, Huntsville, Alabama 35762 \\ S. F. Lyuksyutov ${ }^{\text {a) }}$ and M. A. Reagan \\ Department of Physics, Chemistry, and Polymer Engineering, The University of Akron, Akron, Ohio 44325 \\ P. Buchhave \\ Department of Physics, Technical University of Denmark, Lyngby 2800, Denmark
}

(Received 2 September 2004; accepted 22 November 2004; published online 9 February 2005)

\begin{abstract}
Photogalvanic effect produces actuation of periodic motion of macroscopic $\mathrm{LiNbO}_{3}$ crystal. This effect was applied to the development of an all-optical moving-grating interferometer usable for optical trapping and transport of algae chlorella microorganisms diluted in water with a concentration of $27 \times 10^{4} \mathrm{ml}^{-1}$. (C) 2005 American Institute of Physics. [DOI: 10.1063/1.1850171]
\end{abstract}

\section{INTRODUCTION}

Photogalvanic effect (PGE) or anomalous photovoltaic effect $^{1}$ is a transport phenomenon producing high electric fields $(\sim 100 \mathrm{kV} / \mathrm{cm})$ in ferroelectrics. PGE plays an important role in holographic-grating recording ${ }^{2}$ and was recently demonstrated for optical actuation and as high-voltage power supply. ${ }^{25}$ In this paper we demonstrate PGE generating highvoltage electrical pulses in atmosphere to apply this effect for optical trapping.

Optical trapping is a technique for controlling and manipulating matter on the micro- and nanoscale. Originally suggested for cooling atomic beams, ${ }^{4}$ it has evolved into magneto-optical trapping, ${ }^{5}$ and finally arrived as an irreplaceable tool for optical tweezing and for neutral dielectric microparticles manipulation in $1986 .{ }^{6}$ The technique has produced a major impact in different fields of optics, such as holographic optical tweezing, ${ }^{7,8}$ photonic structures, ${ }^{9}$ and optical trapping in space. ${ }^{10}$ In biology, the concept of optical trapping offers a powerful procedure for manipulating biologically relevant particles, ${ }^{11}$ unzipping DNA molecules, ${ }^{12}$ laser micromanipulation of organic molecules ${ }^{13}$ for medical treatment, ${ }^{14}$ and for environmental cleansing. ${ }^{15}$ The optical field can also be used for microfluidic sorting or arrangement of biological particles. ${ }^{16}$ Thus a paradigm for noncontact control of microorganisms in biological as well as of small particles in the physical sciences has emerged.

A simple method for trapping and transporting particles is through a slow interference pattern (IP) motion formed by two laser beams. Such a trapping was already demonstrated in both photosensitive laser materials ${ }^{17}$ and semiconductors ${ }^{18}$ through photoelectron holes and molecular fragments motion. This trapping (sometimes defined as holographic trapping) of charged particles through the generation of holographic photo-emf (Refs. 19 and 20) is a useful tool for the generation of electric current and for material characterization. ${ }^{19}$ The IP motion can be implemented by introducing a phase shift between two beams in a Mach-

\footnotetext{
${ }^{a)}$ Author to whom correspondence should be addressed; electronic mail: sfl@physics.uakron.edu
}

Zender interferometer, using holographic radial diffraction grating $^{21}$ or electro-optic modulator (EOM). ${ }^{22}$ However, all the existing methods require an additional power source for IP motion.

We introduce theoretically and verify experimentally the conceptually smart interferometer that is suitable for physics and biophysics. An alternative procedure for IP motion, that we suggest, is based on the use of photogalvanic actuator as a mirror in one arm of the interferometer, and does not require an external power source.

An experimental implementation of the interferometer is shown in Fig. 1. It consists of two mirrors, one adjustable photogalvanic actuator, based on photorefractive $z$-cut LN crystal, and a cuvette filled with a biological specimen (shown on inset). The motion of the "smart" mirror produces the running light IP that traps and carries the biological particles inside the cuvette. It has been shown recently that continuous laser illumination of iron-doped (Fe-LN) crystal pro-

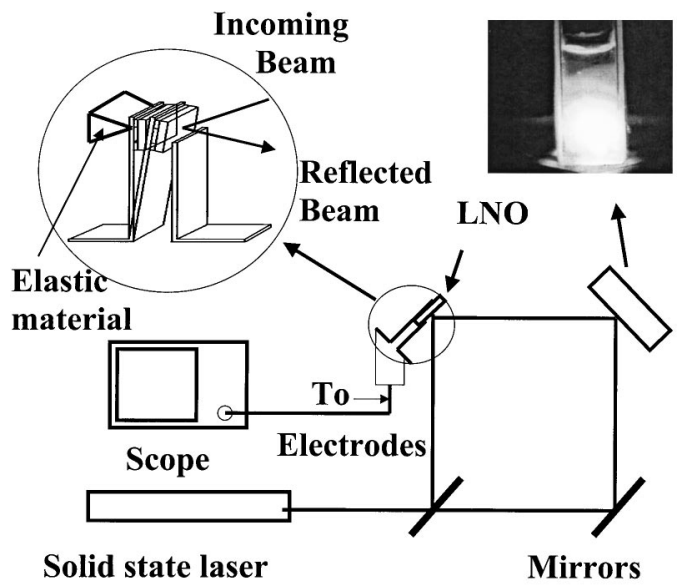

FIG. 1. An experimental implementation of the smart interferometer. A cw solid-state laser at a wavelength of $532 \mathrm{~nm}$ and the intensity varied between 10 and $50 \mathrm{~mW}$ illuminates the LN crystal exciting photogalvanic pulsations at a frequency of $1-10 \mathrm{~Hz}$. Two laser beams produce a running interference pattern at a velocity of $1-10 \mu \mathrm{m} / \mathrm{s}$ in a quartz-made cuvette filled with algae chlorella microorganisms at a concentration of $27 \times 10^{4} \mathrm{ml}^{-1}$ diluted in distilled water. The motion of the interference pattern set the motion of the algae resulting in mass redistribution of the microorganisms shown qualitatively on the inset as a gradient of algae concentration. 


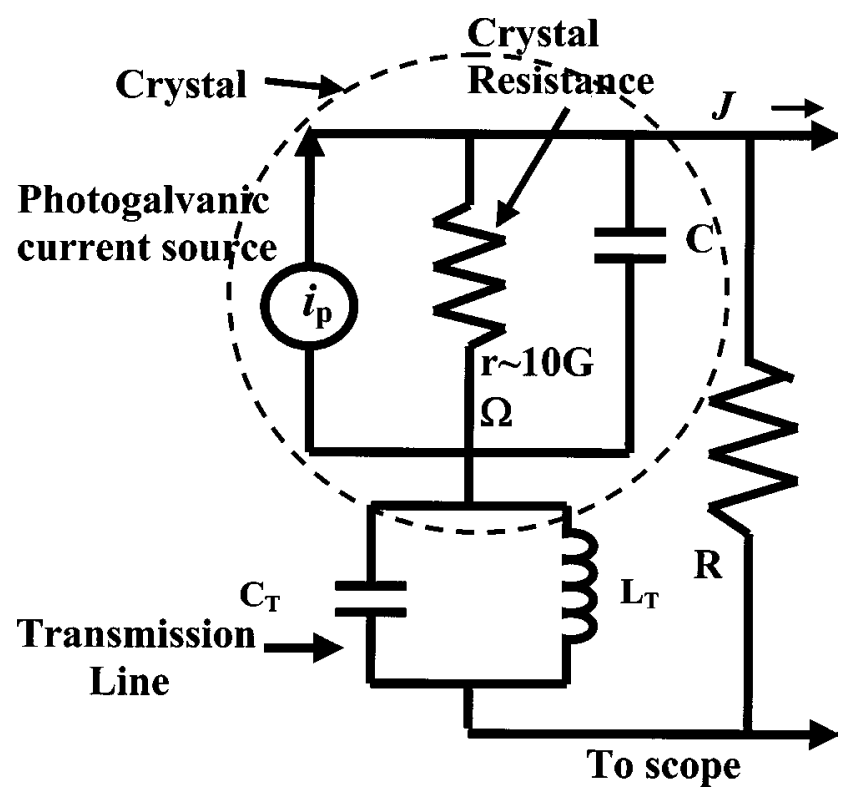

FIG. 2. Equivalent electrical scheme presents the origin of photogalvanic pulsations in an interferometer. A laser illuminates the crystal ("smart mirror") based on lithium niobate. The crystal is presented through the photogalvanic current source $\left(i_{p}\right)$, resistivity $(r \approx 10 \mathrm{G} \Omega)$, and the crystal capacitance $(C)$. A laser illuminates the crystal (encircled) through the hole in the air gap between the grounded electrode and the crystal. The process is modeled through the gas discharge between parallel-plate capacitor.

duces short electrical pulses (up to $100 \mathrm{~V}$ at $1 \mathrm{M} \Omega$ oscilloscope input) as a result of the photogalvanic effect. These pulses induce crystal motion and/or vibrations, thus providing optical actuation. Below, we shall describe the physical principle of photogalvanic actuator and report experimental results related to optical trapping of algae microorganisms.

\section{THEORETICAL PART}

\section{A. Voltage generation from photogalvanic crystal}

The core of the physical model is a smart interferometer based on photorefractive self-adjusted mirror, namely, a photogalvanic actuator. A continuous-wave laser beam of intensity (I) illuminating photorefractive crystal placed between two metal electrodes ("smart mirror") produces a photogalvanic current $\left(i_{p}\right)$ flowing along the $C$ axis of the crystal, which charges the crystal to the potential difference $V_{c}{ }^{22}$ Total electric current $J$, existing within an equivalent electric circuit consisting of the crystal, transmission line, and an external load (shown in Fig. 2), can be written in the following way:

$$
C \frac{d V_{c}}{d t}+i_{p}+\frac{V_{c}}{r}=J .
$$

The first term in this equation represents a displacement current, the second term is associated with photogalvanic current, and the third term is the ohmic current. The sum of these currents constitutes the total external circuit current, which can be measured experimentally. Here, $C$ is the crystal's capacitance, $r$ is the crystal resistance determined by electron concentration $n: r=l / e \mu n S$, where $l$ is the crystal width, $\mu$ is the electron mobility, $S$ is the electrode surface area, and $e$ is the electron charge. The electron density $n$ can be found from the rate equation, ${ }^{20}$

$$
\frac{\partial n}{\partial t}=\left(\beta+s_{0} I\right)\left(N-N_{A}-n\right)-\gamma_{R} n\left(N_{A}+n\right),
$$

where $\beta$ is the thermal generation rate, $s_{0}$ is the photon absorption cross section, $N, N_{A}$ are the concentrations of donor and acceptor centers, respectively, and $\gamma_{R}$ is the recombination constant. Using Kirchhoff's loop theorem, the following equation can be written:

$$
V_{c}+V+J R=0 .
$$

Here $V$ is the voltage drop on the effective transmission line, which includes the air gap between the crystal and one of the electrodes. This set of equations [Eqs. (1)-(3)] belongs to the well-known system of photorefractive equations, ${ }^{19}$ while presenting an adjustment to the band transport model equations. These equations [Eqs. (1)-(3)] describe a voltage difference between the two surfaces of the crystal, which is associated with the photogalvanic current $i_{p}$ leading to the charge separation along the crystal $C$ axis. This charge buildup on the crystal's surfaces creates a condition for the discharge occurring between the crystal and the grounded electrode through the air gap in between them. An additional contribution to the discharge can be associated with local abrupt polarization reversal, ${ }^{23,24}$ but it is not taken into consideration in our model. We therefore assume that the main contribution to the external current is associated with the discharge in the surface air gap.

\section{B. Model of the air gap (discharge gap)}

The current $J$ in the air gap (discharge current) is presented using the model for low-current electric discharge between parallel plane electrodes. ${ }^{25}$ It is assumed that both the electron component $J_{e}$ and positive-ion component $J_{+}$contribute in the net current $J\left(J=J_{e}+J_{+}\right)$. The equation for the electron component $J_{e}$ of the current has a form as

$$
\frac{d J_{e}}{d t}=\frac{J_{p}}{T}+\frac{g(t)-1}{T} J_{e},
$$

where $T$ is the ion transit time, and $g(t)=\gamma\left[(1+\delta) e^{\alpha d}-1\right]$ is the electron number gain. Here, $\alpha$ is the spatial ionization coefficient, $d$ is the thickness of air gap, $J_{p}$ is the photoelectron current emitted from the crystal surface, $\gamma$ is the yield of electrons arriving at the cathode $\left(J_{e}=\gamma J_{+}\right), J_{+}$is the positiveion current, and $\delta$ is the yield of the ions produced by backscattered electrons arriving at the anode. Taking into account that $J_{e}=[\gamma /(1+\gamma)] J$, Eq. (4) may be rewritten as

$$
\frac{d}{d t}\left[\frac{\gamma J}{1+\gamma}\right]=\frac{J_{p}}{T}+\frac{g(t)-1}{T}\left(\frac{\gamma J}{1+\gamma}\right) .
$$

Following Ref. 25 we shall further assume that the yield $(\gamma)$ can be written in the form $\gamma=\gamma_{p}+k_{v} V+k_{l} J$ (8). Where $\gamma_{p}$ is the "potential ejection" yield of electrons, $k_{v}$ is the coefficient describing "kinetic" electron contribution, and $k_{l}$ is the coefficient related to the space-charge field formation. Now, Eq. (5) may be transformed into the following: 


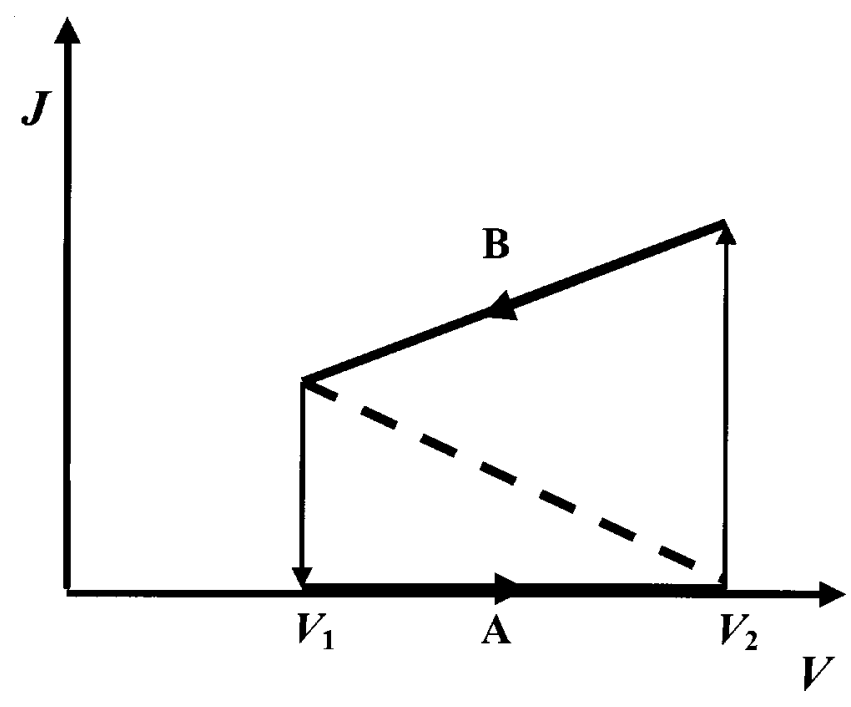

FIG. 3. $S$-type $J$ - $V$ dependence with two branches corresponds to branch "A" or "charging" from the voltage $V_{1}$ to $V_{2}$, and branch "B" or "discharging" from the voltage $V_{2}$ to $V_{1}$.

$$
\begin{aligned}
\frac{d J}{d t}= & \left(\frac{(1+\gamma)}{\gamma} \frac{J_{p}}{T}+\frac{J}{(1+\gamma)}\left\{\frac{[g(t)-1](1+\gamma)}{T}-\frac{k_{v} d V}{\gamma d t}\right\}\right) \\
& \times\left[1+\frac{k_{l} J}{\gamma(1+\gamma)}\right]^{-1} .
\end{aligned}
$$

Eliminating $V_{c}$ from (3) we can rewrite (1) as

$$
\frac{d V}{d t}+R \frac{d J}{d t}=\frac{i_{p}}{C}-\frac{1}{r C}[V+J(R+r)] .
$$

This system of equations [Eqs. (6) and (7)] is similar to that considered in Ref. 25. The set describes pulsating responses for current $J$ and discharge voltage $V$. The spatial ionization coefficient $\alpha$ can be presented as

$$
\alpha=\frac{A}{d} \exp \left(-\frac{V_{0}}{V}\right)\left[(a V)^{1.5}+1\right]^{1 / 2},
$$

with adjustable free parameters $A, V_{0}$, and $a$. Small signal analysis of Eqs. (6) and (7) indicates the existence of a negative differential resistance leading to oscillatory responses including periodic pulsations of the current and the voltage. ${ }^{25}$ Here we suggest a simple analytical approach that is applicable for such dynamic systems and adjusted for treating pulsating solutions.

\section{ANALYTICAL APPROACH}

The system of Eqs. (6) and (7) can be solved numerically. However, for a qualitative consideration or first order approximation, we apply an analysis used for systems exhibiting properties of nonlinear dynamics. We assume that the $J-V$ dependence of the equivalent electric scheme shown in Fig. 2 has an "S-like" shape (Fig. 3) with a negative differential resistance. We approximate the $J$ - $V$ dependence by two branches. The first branch shown in Fig. 3 as "A" corresponds to $J=0$ or to the "off" (crystal charging) state of the system. The branch two, shown as "B," corresponds to $J$ $=\left(V-V_{i}\right) / R_{i}$ and is the "on" (crystal discharging) state. Combining Eq. (7) with $J$ given above, we obtain the solution for
$V$ for both the states. $V_{i}$ and $R_{i}$ are the effective voltage and resistance, respectively. For the charging branch "A,"using initial condition $V(t=0)=V_{1}$, the solution of Eq. (7) can be presented as,

$$
V(t)=i_{p} r+\left(V_{1}-i_{p} r\right) e^{-t / \tau_{1}},
$$

where $\tau_{1}=r C$, and the charging time $T_{1}$ needed to reach voltage $V_{2}$ is

$$
T_{1}=\tau_{1} \ln \left(\frac{V_{1}-i_{p} r}{V_{2}-i_{p} r}\right) .
$$

For the discharging branch "B," $\left(t \geqslant T_{1}\right)$, the solution of Eq. (7) yields

$$
V=D+\left(V_{2}-D\right) e^{\left(T_{1}-t\right) / \tau_{2}}
$$

with corresponding discharge time

$$
T_{2}-T_{1}=\tau_{2} \ln \left(\frac{V_{2}-V_{i}-B}{V_{1}-V_{i}-B}\right),
$$

where

$$
\begin{aligned}
& \tau_{2}=\frac{\tau_{1}\left(1+R R_{i}^{-1}\right)}{1+(R+r) R_{i}^{-1}}, \quad D=\left[\frac{i_{p} r+V_{i}(R+r) / R_{i}}{1+(R+r) / R_{i}}\right], \\
& B=\left\{\frac{r i_{p}-V_{i}}{[1+(R+r)] / R_{i}}\right\} .
\end{aligned}
$$

From this analytical approach the period of oscillation $T$ is defined as

$$
T_{2}=\tau_{1} \ln \left(\frac{V_{1}-i_{p} r}{V_{2}-i_{p} r}\right)+\tau_{2} \ln \left(\frac{V_{2}-V_{i}-B}{V_{1}-V_{i}-B}\right) .
$$

The solution of Eq. (7) is presented in Fig. 4(a) for free parameter values: $i_{p}=10^{-8} \mathrm{~A}, r=10^{12} \Omega, C=10^{-12} \mathrm{~F}, R$ $=10^{6} \Omega, R_{i}=10^{7} \Omega, V_{1}=100 \mathrm{~V}, V_{2}=10^{3} \mathrm{~V}$, and $V_{i}=50 \mathrm{~V}$. Figure 4(b) shows the dependence of the discharge voltage and current in time that is proportional to the experimental data on voltage pulsations detected by an oscilloscope, as shown in Fig. 4(b). To fit the theoretical value of the maximal current $\left(\approx 10^{-4} \mathrm{~A}\right)$, we needed to assume an effective resistance of the oscilloscope and transmission line to be $10^{4} \Omega$.

By this dynamic-system-based approach, we are able to find the period of oscillations and approximate time dependence of both $V$ and $J$. This allows the Fourier spectral analysis to find the spectral range of the observed signal. The existence of pulsations was also confirmed using numerical calculations of similar equations in Ref. 25.

\section{EXPERIMENT}

$\mathrm{A} \mathrm{LNbO}_{3}: \mathrm{Fe}(0.05 \%)$ crystal is placed in one arm of the interferometer. The crystal is attached to a thin flat copper electrode. Another electrode is located in front of the crystal approximately 1-2 $\mathrm{mm}$ away from the front of the crystal. This configuration represents an asymmetric capacitor. A solid-state $\mathrm{cw}$ laser radiating at $532 \mathrm{~nm}$ impinges onto the crystal causing the crystal to develop a negative charge buildup on its face, which is opposite to the $C$ axis. Surface 

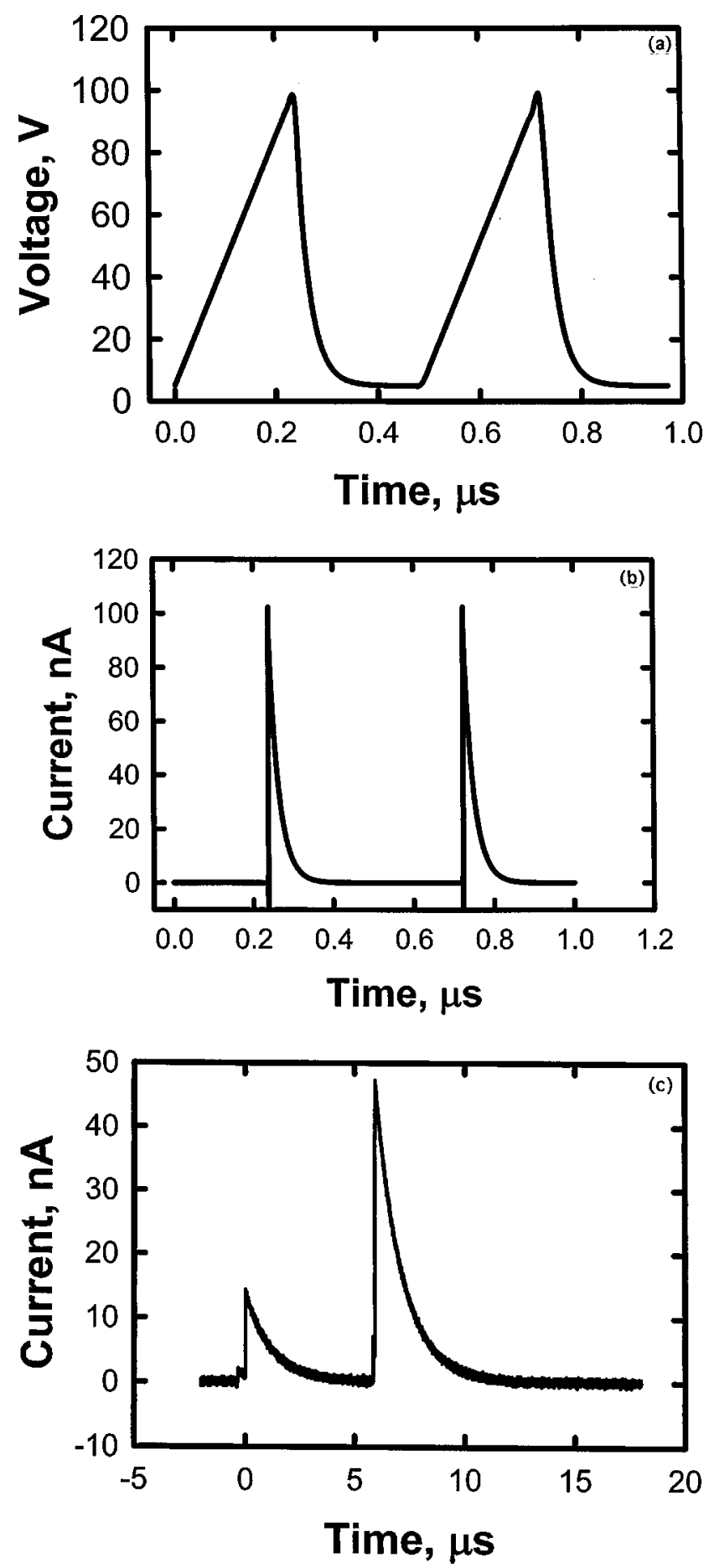

FIG. 4. The results of modeling are shown as (a) voltage time and (b) current in the external circuit on time dependencies. To fit the theoretical value for current, we need to assume the effective resistance of the oscilloscope and transmission line as $10^{4} \Omega$; (c) experimentally measured voltage pulses from the screen of an oscilloscope as a function of time in an equivalent electric scheme.

charge builds up on the opposite face of the crystal, causing the crystal and the attached electrode to be drawn towards the other electrode by electrostatic attraction. As the crystal moves, the reflected beam deflected which causes the interference pattern to move when recombined with the stationary reference beam. Once the crystal becomes close enough to the other electrode, there is a discharge and the crystal moves quickly back to a vertical position. A stationary piece of an elastic material was located just behind the electrode that was attached to the crystal to prevent vibrations as the crystal discharged and returned to a vertical position. This process occurs cyclically and can be monitored through voltagecurrent pulsations on the Hewlett-Packard oscilloscope and through the motion of the interference pattern associated with crystal vibrations. This mechanical motion of the macroscopic LN crystal (weight is $0.47 \mathrm{~g}$ ) was actuated by the low-power solid-state $\mathrm{cw}$ laser $(P=100 \mathrm{~mW}, \lambda=532 \mathrm{~nm}){ }^{3}$ The interference pattern motion is subsequently actuated by the photogalvanic electric field. Experimentally detected voltage and current pulsations have a saw-tooth shape. The pulsations slope determines the speed (in the range $1-10 \mu \mathrm{m} / \mathrm{s}$ ) of the running interference pattern and it grows with the laser intensity varied between 10 and $50 \mathrm{~mW}$. This allows us to tune the pattern's speed simply by adjusting the laser intensity. An adequate response (increase) of the interference pattern speed developing with the laser intensity may be considered as a smart behavior of a photomechanical interferometer. Relatively smooth motion of the interference pattern, with the speed developing with intensity (laser power was $100 \mathrm{~mW}$ ), is an additional indication on the correctness of our model. To confirm that this interferometer is an effective optical trapping device, we used a water solution of algae chlorella at the concentration of $27 \times 10^{4} \mathrm{ml}^{-1}$. The algae was placed in a quartz cuvette $\left(10 \times 10 \times 50 \mathrm{~mm}^{3}\right)$ and illuminated by the interference pattern from the green solidstate laser. We have compared the quality of running-grating patterns, created by the smart interferometer, with those received with a rotating radial grating. ${ }^{21}$ The quality of the patterns were comparable, but the actual experimental results with algae were done with the rotating radial grating to ensure better quality of the results in the stable and longrunning experiment.

The device additionally suggests an actuation so that the motion of the LN crystal due to photogalvanic pulsations controls the motion of the interference pattern. It has been shown that the LN crystal of a 470-mg weight was displaced at an angle of $2^{\circ}$ through photogalvanic pulsations. The displacement rate of the crystal determines the time scale IP to move.

\section{SUMMARY}

We theoretically predicted and experimentally confirmed the feasibility of the smart photomechanical interferometer. The interferometer is based on the photogalvanic effect in the LN crystal placed in one of the arms of Mach-Zender interferometer. This interferometer creates a slow-moving interference pattern with the motion speed increasing with the laser intensity, "smart behavior." No external voltage function generator is needed for the operation of this device. It was experimentally confirmed that this compact interferometer uses a $100-\mathrm{mW} \mathrm{cw}$ laser producing sufficient power for the generation of self-regulated running interference pattern for optical trapping of algae microorganisms. Thus the device developed in this work can have a double physical ap- 
plication, an actuator controlling the motion of the IP through photogalvanic pulsations and an interferometer trapping and moving microorganisms.

\section{ACKNOWLEDGMENTS}

Partial support of this research through the NSF Doctoral Capacity Building Grant No. 0236425, NASA Grant No. NAG5-12028, and HBCU/MI Environmental Technology Consortium are gratefully acknowledged. Also, we thank Dr. Tadros of AAMU Biology Department for supplying the algae for optical trapping test, and Jason Bowling of UA for technical assistance.

${ }^{1}$ A. M. Glass, D. Von der Linde, and T. J. Negran, Appl. Phys. Lett. 25, 233 (1974)

${ }^{2}$ N. Kukhtarev, S. Lyuksyutov, P. Buchhave, T. Kukhtareva, K. Sayano, and P. Banerjee, Phys. Rev. A 58, 4051 (1998).

${ }^{3}$ N. Kukhtarev et al., J. Nonlinear Opt. Phys. Mater. 11, 203 (2002).

${ }^{4}$ A. Ashkin, Phys. Rev. Lett. 40, 729 (1978).

${ }^{5}$ H. Metcalf and P. van der Straten, Phys. Rep. 244, 203 (1994).

${ }^{6}$ A. Ashkin, J. M. Dziedzic, J. E. Bjorkholm, and S. Chu, Opt. Lett. 11, 288 (1986).

${ }^{7}$ P. Korda, G. Spalding, E. R. Dufresne, and D. G. Grier, Rev. Sci. Instrum. 73, 1956 (2002).

${ }^{8}$ J. E. Curtis, B. A. Koss, and D. G. Grier, Opt. Commun. 207, 169 (2002).
${ }^{9}$ R. Holmlin, M. Schiavoni, and G. Whitesides, Angew. Chem., Int. Ed. 39, 3503 (2001).

${ }^{10}$ K. Taguchi, M. Tanaka, and M. Ikeda, Opt. Rev. 8156 (2001).

${ }^{11}$ C. L. Kuyper and D. T. Chiu, Appl. Spectrosc. 56, 300A (2002).

${ }^{12}$ U. Bockelmann, P. Thomen, B. Essevaz-Roulet, V. Viasnoff, and F. Heslot, Biophys. J. 82, 1537 (2002).

${ }^{13}$ K. Schutze, H. Posl, and G. Lahr, Cell Mol. Biol. (Paris) 44, 735 (1998).

${ }^{14}$ K. O. Greulich and G. Pilarczyk, Cell Mol. Biol. (Paris) 44, 701 (1998).

${ }^{15}$ N. V. Kukhtarev, T. Kukhtareva, E. Ward, J. Jones, and H. J. Caulfield, Opt. Eng. 37, 2597 (1998).

${ }^{16}$ M. P. MacDonald, G. C. Spalding, and K. Dholakia, Nature (London) 426, 421 (2003).

${ }^{17}$ N. Noginova, N. V. Kukhtarev, B. S. Chen, H. J. Caulfield, P. Venkateswarlu, and M. Noginov, J. Opt. Soc. Am. B 13, 1 (1996).

${ }^{18}$ S. Mansurova, S. Stepanov, N. Korneev, and C. Dibon, Opt. Commun. 152, 207 (1998).

${ }^{19}$ N. V. Kukhtarev, T. Kukhtareva, and P. Buchhave, Photorefractive Materials and Applications (Academic, New York, 2000), p. 1.

${ }^{20}$ V. Vinetskii and N. Kukhtarev, Fiz. Tverd. Tela (Leningrad) 16, 3714 (1975) Sov. Phys. Solid State 16, 2414 (1975)].

${ }^{21}$ P. Buchhave, S. Lyuksyutov, and M. Vasnetsov, Opt. Lett. 20, 2363 (1995).

${ }^{22}$ M. Arlt, M. P. McDonald, L. Paterson, W. Sibbett, K. Dholakia, and K. Volkesepulveda, Opt. Express 10, 844 (2002).

${ }^{23}$ G. Roseman, D. Shur, Y. Krasic, and A. Dunaevsky, Appl. Phys. Lett. 88, 6109 (2000).

${ }^{24}$ H. Riege, J. Boscola, J. Handerek, and V. Herleh, J. Appl. Phys. 84, 1602 (1998).

${ }^{25}$ A. Phelps, Z. Petrovic, and B. Jelenkovic, Phys. Rev. E 47, 2825 (1993). 